\title{
Wnt signaling control of bone cell apoptosis
}

\author{
Peter V N Bodine ${ }^{1}$ \\ ${ }^{1}$ Women's Health and Musculoskeletal Biology, Wyeth Research, Collegeville, Pennsylvania 19426, USA
}

\begin{abstract}
Wnts are a large family of growth factors that mediate essential biological processes like embryogenesis, morphogenesis and organogenesis. These proteins also play a role in oncogenesis, and they regulate apoptosis in many tissues. Wnts bind to a membrane receptor complex comprised of a frizzled (FZD) G-protein-coupled receptor and a low-density lipoprotein (LDL) receptor-related protein (LRP). The formation of this ligand-receptor complex initiates a number of signaling cascades that include the canonical/beta-catenin pathway as well as several noncanonical pathways. In recent years, canonical Wnt signaling has been reported to play a significant role in the control of bone formation. Clinical studies have found that mutations in LRP-5 are associated with reduced bone mineral density (BMD) and fractures. Investigations of knockout and transgenic mouse models of Wnt pathway components have shown that canonical Wnt signaling modulates most aspects of osteoblast physiology including proliferation, differentiation, function and apoptosis. Transgenic mice expressing a gain of function mutant of LRP-5 in bone, or mice lacking the Wnt antagonist secreted frizzled-related protein-1, exhibit elevated BMD and suppressed osteoblast apoptosis. In addition, preclinical studies with pharmacologic compounds such as those that inhibit glycogen synthase kinase- $3 \beta$ support the importance of the canonical Wnt pathway in modulation of bone formation and osteoblast apoptosis.
\end{abstract}

Keywords: LDL receptor-related protein, secreted frizzled-related protein, glycogen synthase kinase, osteoblast, bone formation, programmed cell death

Cell Research (2008) 18:248-253. doi: 10.1038/cr.2008.13; published online 22 January 2008

\section{Wnt signaling}

Wnts are a large family of 19 secreted carbohydrateand lipid-modified proteins that mediate vital biological processes like embryogenesis, organogenesis and tumorigenesis [1-4]. These proteins bind to a membrane receptor complex composed of a Frizzled (FZD) G-protein coupled receptor and a low-density lipoprotein (LDL) receptorrelated protein (LRP) [2-4]. There are 10 different FZDs as well as 2 LRPs (5 and 6), and ligand binding activates one of several intracellular signaling pathways depending upon the Wnt, FZD receptor and cell-type involved [1-4]. The best characterized of these is the canonical or Wnt/ $\beta$-catenin pathway that signals through LRP-5 or LRP-6 and initiates activation of disheveled (Dsh), which leads to inhibition of glycogen synthase kinase (GSK)-3 $\beta$ and subsequent stabilization of $\beta$-catenin. The stabilized $\beta$-catenin protein then translocates to the nucleus, where

Correspondence: Peter V N Bodine

Tel: +484-865-2717; Fax: +484-865-9368

E-mail: bodinep@wyeth.com it binds to and activates lymphoid-enhancer binding factor (LEF)/T cell-specific transcription factors (TCFs) that are members of the high-mobility-group (HMG)-box transcription factor family [1-5]. Additional non-canonical pathways are also activated by Wnts $[2,6]$. These include the G-protein mediated Wnt/calcium pathway [7] and the c-Jun $\mathrm{NH}_{2}$-terminal kinase (JNK) pathway [8], both of which can be controlled by Dsh [6]. Recently, Wnts have also been shown to stimulate adenylyl cyclase and increase cAMP levels via a G-protein coupled mechanism $[9,10]$. Although the importance of the canonical pathway in the control of fundamental mammalian processes is thoroughly established, the significance of the non-canonical pathways is less well understood.

Due to the importance of Wnt signaling in the control of key biological processes, it is not surprising that many extracellular and intracellular regulators of the pathways exist [1-3]. The extracelluar regulators include a variety of secreted proteins like Wnt inhibitory factors (WIFs), secreted frizzled-related proteins (SFRPs), dickkopfs (DKKs) [11-13] as well as SOST/sclerostin, Wise and connective tissue growth factor (CTGF) [14, 15]. These proteins bind Wnts (WIFs and SFRPs) or FZD receptors (SFRPs), or 
interact with LRPs (DKKs, SOST, Wise and CTGF).

\section{Wnt signaling control of apoptosis}

Activation of canonical Wnt signaling has been associated with either a decrease or increase in apoptosis $[16,17]$. Chen et al. reported that stable expression of Wnt-1 in Rat-1 cells suppressed apoptosis induced by either vincristine or vinblastine [18]. Suppression of apoptosis by Wnt-1 was demonstrated using assays such as DNA fragmentation, annexin V staining, cytochrome c release and caspase-9 activation and did not involve regulation of JNK or Akt pathways. However, stable expression of a dominant negative TCF blocked the reduction of programmed cell death (PCD) by Wnt-1, demonstrating that canonical signaling was involved in this process.

Melkonyan et al. identified a family of factors, which they termed secreted apoptosis-related proteins (SARPs) [19]. These proteins, SARP-1, -2 and -3 , are more commonly known as the Wnt antagonists SFRP-2, -1 and -5 , respectively. Stable expression of SARP-1/SFRP-2 in MCF-7 human breast adenocarcinoma cells reduced the cytotoxic effects of TNF- $\alpha$ and ceramide, while expression of SARP-2/SFRP-1 sensitized the cells to these apoptotic agents. The ability of these secreted proteins to modulate cell death correlated with control of canonical Wnt signaling. Expression of SARP-1/SFRP-2 in MCF-7 cells increased $\beta$-catenin protein levels, while expression of SARP-2/SFRP-1 had the opposite effect and reduced $\beta$-catenin.

Although in MCF-7 cells SFRP-1 accelerates apoptosis induced by cytotoxic agents, this Wnt antagonist has the opposite effect on human periodontal ligament fibroblasts (PDLFs). Han and Amar showed that SFRP-1 mRNA and protein levels were high in PDLFs, but were low in human gingival fibroblasts (GFs) [20]. Ceramide treatment of PDLFs induced SFRP-1 expression, while this cytotoxic agent had no effect on SFRP-1 production in GFs. Co-treatment of GFs with ceramide and SFRP-1 protein increased viability and reduced apoptosis, while the addition of SFRP-1 had no effect on PDLFs, which already expressed the protein and were resistant to ceramide treatment. Conversely, treatment of PDLFs with an siRNA to SFRP-1 sensitized the cells to ceramide, while the siRNA had no effect on GFs, which did not express the protein. Thus, the ability of SFRP-1 to regulate apoptosis is cell context dependent.

\section{Bone remodeling}

Bone is formed and maintained by teams of osteoblasts and osteoclasts working together in a coordinated manner.
This process is known as remodeling, which is the mechanism for replacing immature, damaged or aged bone with new lamellar bone $[21,22]$. Osteoblasts are bone-forming cells that synthesize and mineralize the skeleton [23, 24], while osteoclasts are the cells that resorb the calcified tissue [25].

Osteoblasts develop from bone marrow-derived multipotent mesenchymal stem cells of the colony forming unit-fibroblast (CFU-F) lineage that also give rise to fibroblasts, myoblasts, adipocytes, and chondrocytes [23, 24]. The earliest osteoprogenitor cells express the bonespecific transcription factor Runx2, which is required for differentiation of these cells into mature osteoblasts [26, 27]. Osteoprogenitors undergo further differentiation to pre-osteoblasts that still proliferate [23, 24], and these cells then develop into mature osteoblasts that no longer divide, but instead synthesize the bone matrix [23, 24]. Mature osteoblasts have one of three fates upon completion of the synthetic phase of the remodeling cycle [23, 24]: they can become osteocytes upon entrapment within the mineralized matrix; they can evolve into inactive lining cells that protect the bone matrix from osteoclasts; or they can undergo apoptosis, which appears to be the fate of up to $80 \%$ of the cells $[21,28]$. The role of apoptosis in this process is probably to eliminate excess osteoblasts that are not needed as either lining cells or osteocytes. Some of the key proteins that osteoblasts express are type I collagen (TIC), alkaline phosphatase (ALP), bone sialoprotein (BSP) and osteocalcin (OC) [23, 24].

Osteoclasts are multinucleated giant cells that originate from bone marrow-derived hematopoietic stem cells of the monocyte/macrophage lineage [25]. These cells produce resorption pits when attached to bone and express important marker proteins such as the calcitonin receptor, tartrate-resistant acid phosphatase and the vitronectin receptor [23]. The earliest identifiable progenitor of the osteoclast is the granulocyte and macrophage colony-forming unit (CFUGM) cell. When these cells bind the cytokines receptor activator of nuclear factor- $\mathrm{\kappa B}$ ligand (RANKL), colony stimulating factor (CSF)-1/monocyte-colony stimulating factor (M-CSF), and either interleukin (IL)-1 or tumor necrosis factor (TNF)- $\alpha$, they undergo differentiation and subsequent fusion to become mature multinucleated osteoclasts [25]. Of these factors, RANKL and CSF-1/M-CSF are thought to be essential for this process. Both of these cytokines are expressed by bone marrow stromal cells (BMSCs) and osteoblasts, and at least part of the osteoclastogenic activities of IL- 1 and TNF- $\alpha$ are mediated by up-regulation of RANKL and CSF-1/M-CSF in these cells. Consequently, BMSCs and osteoblasts are thought to be required for the differentiation and activity of osteoclasts [21]. In addition to expressing bone-resorbing cytokines 
such as RANKL, cells of the osteoblast lineage also produce osteoclastogenic antagonists like osteoprotegerin (OPG). OPG is a soluble TNF- $\alpha$ receptor that binds to and blocks the ability of RANKL to signal to osteoclast lineage cells [25].

\section{Role of apoptosis in bone cell physiology and phar- macology}

Many physiologic and pharmacologic agents that affect bone remodeling do so in part by controlling bone cell apoptosis [28]. Estrogens and bisphosphonates induce osteoclast PCD but have the opposite effect on osteoblasts and osteocytes, which may partly explain the ability of these agents to suppress bone turnover in diseases like osteoporosis. On the other hand, intermittent treatment with PTH suppresses apoptosis of osteoclasts, osteoblasts and osteocytes. Pharmacologic (i.e., micromolar) levels of glucocorticoids do not appear to control osteoclast PCD, but induce apoptosis of osteoblasts and osteocytes, and this is thought to be the mechanism by which these steroids cause bone loss. Androgens also do not appear to regulate osteoclast apoptosis, but suppress the death of osteoblasts and osteocytes. Similarly, other bone anabolic agents like insulin-like growth factors (IGFs) reduce osteoblast and osteocyte PCD.

\section{Regulation of bone formation by canonical Wnt signaling}

The first evidence for the involvement of canonical Wnt signaling in bone formation came from human genetic studies of osteoporosis pseudoglioma (OPPG) syndrome and high bone mass (HBM) phenotypes [29, 30]. Gone et al. [31] reported that numerous nonsense and frame-shift mutations throughout the coding region of the human Wnt co-receptor LRP-5 cause OPPG syndrome. These loss-offunction mutations lead to dramatic reductions in trabecular bone volume (TBV), premature fractures and skeletal deformities that resulted from diminished bone accrual. In addition, developmental eye defects also occurred from the loss of LRP-5 function [31].

On the other hand, a gain-of-function mutation in LRP-5 has the opposite effect and causes a HBM trait in humans [32-34]. Two groups, Little et al. [35] and Boyden et al. [36], found the same G171V mutation in two families that had HBM. This mutation resides in the first $\beta$-propeller motif of the extracellular domain of LRP-5 and leads to an increase in hip and spine aerial bone mineral density (aBMD) as determined by dual-energy X-ray absorptiometry (DEXA). Radiographs showed that the bones of HBM individuals have increased trabecular BMD and cortical thickness but are otherwise normal in shape [37]. Additional gain-of-function mutations of human LRP-5 (G171R, A214T, A214V, A242T, T253I and D111Y) also lead to HBM phenotypes like endosteal hyperostosis, Van Buchem disease, autosomal dominant osteosclerosis and osteopetrosis type I [38].

An interesting observation is that all of the gain-offunction mutations identified to date that cause HBM phenotypes are found in the first $\beta$-propeller domain of LRP-5 [39]. These mutations are scattered throughout the 6 blades of the $\beta$-propeller motif, and this leads to relief from DKK-1 antagonism [36]. DKK-1 binds to the $3^{\text {rd }}$ and $4^{\text {th }} \beta$-propeller motifs of LRP-5 and LRP- 6 and disrupts canonical signaling by inducing LRP internalization and degradation through kremen $[13,39]$. Another secreted Wnt antagonist, SOST/sclerostin, has been reported to bind to and antagonize LRP-5 and LRP-6 by interacting with the receptors' $1^{\text {st }}$ and $2^{\text {nd }} \beta$-propeller motifs [40-42].

\section{Role of wnt signaling in the control of bone cell apoptosis}

LRP-5 knockout mice and transgenic mouse models of LRP-5 mutations have allowed us to understand the mechanisms by which canonical Wnt signaling controls bone formation. Kato et al. showed that deletion of murine LRP-5 reduced vertebral trabecular bone volume (TBV) when compared to LRP $-5^{+/+}$controls as determined by histomorphometry [43]. LRP- $5^{-/}$mice also had tibial fractures as assessed by radiographs. Dynamic histomorphometric analysis of the vertebrae from LRP $-5^{-/}$mice demonstrated that deletion of LRP-5 decreased the mineral apposition rate (MAR) indicating that osteoblast function was inhibited by the loss of the gene. Furthermore, deletion of LRP-5 reduced osteoblast numbers in long bones, and this was correlated with a decrease in calvarial osteoblast proliferation as determined by bromodeoxyuridine (BrdU) labeling. However, osteoblast apoptosis and differentiation were not altered by loss of LRP-5.

In contrast to the osteopenic/osteoporotic phenotype of the LRP-5 knockout mice [43], our group showed that LRP-5 gain-of-function transgenic mice have HBM [44]. These animals were developed by targeted expression of human LRP- $5^{\mathrm{G} 171 \mathrm{~V}}$ in bone using the $3.6 \mathrm{~kb}$ rat type I collagen promoter. The transgenic mice have increased bone formation, but the mechanisms for this effect are different from those that lead to decreased bone formation by the loss of LRP-5. Heterozygous mice (LRP- $5^{\mathrm{G} 171 \mathrm{~V} /+}$ ) have increased distal femur trabecular volumetric BMD (vBMD) as measured by peripheral quantitative computed tomography (pQCT). In addition, cortical bone thickness is also increased in the LRP-5 ${ }^{\mathrm{G} 171 \mathrm{~V} /+}$ mice. Histological analysis 
of the femurs indicated that total bone area is increased in the transgenic mice, while the mineralizing surface is also elevated. However, MAR is not significantly increased in the LRP- $5^{\mathrm{G} 171 \mathrm{~V} / \mathrm{+}}$ mice, indicating that osteoblast activity is not affected by the mutation. ALP staining of calvaria is also elevated in the LRP- $5^{\mathrm{G} 171 \mathrm{~V} / \mathrm{t}}$ mice, while TUNEL (terminal dNTP transferase-mediated dUTP nick end-labeled) staining demonstrated that osteoblast and osteocyte apoptosis is reduced. Thus, the primary mechanism for increased bone formation in the LRP- $5^{\mathrm{G} 171 \mathrm{~V} /+}$ mice appears to be elevated osteoblast/osteocyte numbers due to decreased cell death, which indicates that modulation of this process can have a significant impact on the skeleton [28].

We discovered that SFRP-1 plays a role in osteoblast physiology during a series of transcription profiling experiments that sought to elucidate the molecular events associated with human osteoblast differentiation and bone formation [45]. Basal SFRP-1 mRNA levels were observed to increase during human osteoblast (HOB) differentiation from pre-osteoblasts to pre-osteocytes, and then decline in mature osteocytes. This expression pattern correlated with levels of cellular viability such that the pre-osteocytes, which had the highest levels of SFRP-1 mRNA, also had the highest rate of cell death. In addition, expression of SFRP-1 mRNA was induced following PGE $_{2}$ treatment of pre-osteoblasts and mature osteoblasts that have low basal SFRP-1 message levels. In contrast, SFRP-1 expression was observed to be down-regulated following transforming growth factor (TGF)- $\beta 1$ treatment of pre-osteocytes that have high basal SFRP-1 mRNA levels. Consistent with this observation, treatment of pre-osteoblasts and mature osteoblasts with $\mathrm{PGE}_{2}$ increased apoptosis, while treatment of pre-osteocytes with TGF- $\beta 1$ decreased cell death. Likewise, over-expression of SFRP-1 in HOB cells that express low levels of the gene product accelerated the rate of cell death. Together, these results implied that SFRP-1 is key modulator of human osteoblast and osteocyte survival.

In order to confirm these in vitro observations, we characterized a knockout mouse model [46]. These mice expressed the $L a c Z$ gene in place of exon 1 of SFRP-1 so that SFRP-1 promoter activity could be measured by bgalactosidase staining. Loss of SFRP-1 in mice increased distal femur TBV as determined by micro-CT. But in contrast to the LRP- $5^{\mathrm{G} 171 \mathrm{~V} /+}$ mice, loss of SFRP-1 had no effect on cortical bone parameters. An interesting observation about the SFRP- $1^{-/}$mice was that prior to 13 weeks of age, there was no difference in trabecular vBMD of the distal femur as determined by $\mathrm{pQCT}$ between wild-type and knockout mice. However, as the mice aged, the SFRP- $1^{+/+}$ animals lost trabecular bone, while the SFRP $-1^{-/}$mice gained trabecular bone. Thus, deletion of SFRP-1 delays and enhances the onset of peak bone mass and suppresses senile bone loss.

As with the LRP- $5^{-/-}$mice, deletion of SFRP-1 also affected osteoblast activity. Dynamic histomorphometric analysis of proximal femurs from SFRP- $1^{+/+}$and SFRP$1^{-/}$female mice showed that deletion of SFRP-1 increased the mineral apposition rate, indicating that osteoblast activity was increased by loss of the gene. In addition, like the LRP- $5^{\mathrm{G} 171 \mathrm{~V} / \mathrm{+}}$ mice, deletion of SFRP-1 also suppressed apoptosis. TUNEL staining of calvaria from female mice demonstrated that loss of SFRP-1 led to an increase in calvarial thickness and a decrease in osteoblast and osteocyte PCD. In contrast to the LRP- $5^{-1-}$ mice, deletion of SFRP-1 also affects osteoblast differentiation. When bone marrow from SFRP- $1^{+/+}$and SFRP- $1^{-/-}$female mice was differentiated to osteoblasts in culture by incubation with ascorbic acid, $\beta$-glycerolphosphate and dexamethasone, the number of ALP+ cells was increased by deletion of SFRP-1. Analysis of the differentiating cultures for LacZ expression showed that osteoblast development and matrix mineralization also increased as SFRP-1 promoter activity became elevated, suggesting that control of Wnt signaling by SFRP-1 modulates osteoblast differentiation and function. In addition, evaluation of the bone marrow cultures from knockout mice by TUNEL staining showed that cellular apoptosis was suppressed when compared to cultures from wild-type controls. Finally, like the LRP- $5^{-/}$mice, deletion of SFRP-1 also affects osteoblast proliferation. Measurement of DNA synthesis in cultures derived from calvaria of newborn SFRP- $1^{+/+}$and SFRP- $1^{-/-}$mice showed that osteoblast proliferation increased in the knockout cells during the proliferative-phase. However, when the SFRP$1^{-/}$cultures reached confluence and proliferation ceased, the rate of DNA synthesis returned to normal, indicating that the proliferation-differentiation transition was not altered by loss of SFRP-1. Therefore, deletion of SFRP-1 enhances osteoblast proliferation, differentiation and function, and suppresses osteoblast and osteocyte apoptosis.

Consistent with a negative effect of SFRP-1 on osteoblasts and osteocytes, Wang et al. reported that pharmacologic doses of glucocorticoids also increase osteoblast SFRP-1 expression and decrease bone formation in rats [47]. These authors showed that high concentrations $\left(\geq 10^{-6} \mathrm{M}\right)$ of dexamethasone up-regulated SFRP-1 mRNA and protein levels in primary rat MSCs in vitro, and that this correlated with suppression of osteogenesis, reduced cytosolic $\beta$-catenin protein levels and blunted nuclear Runx2 activation, as well as increased apoptosis. Steroid treatment of rats in vivo increased SFRP-1 protein levels in trabecular osteoblasts and osteocytes, and this correlated with increased bone cell apoptosis as well as decreased femoral TBV and bone strength. Confirmation that SFRP-1 was involved in these processes was obtained from experi- 
ments using siRNA in vitro as well as purified proteins in vitro and in vivo.

Additional evidence that Wnts prevent osteoblast apoptosis in vitro was recently reported by Ameida et al. [48]. Using murine $\mathrm{C} 2 \mathrm{C} 12$, OB-6 and MC-3T3-E1 cells, the authors showed that treatment with both canonical (Wnt3a) and noncanonical (Wnt-5a) Wnts suppressed PCD. In addition, while canonical Wnt signaling appeared to play a role in controlling cell survival, additional signaling pathways like Src/Erk (extracellular signal-regulated kinase) and PI3K (phosphatidylinositol 3-kinase)/AKT were also involved in this process.

More recently, Tobimatsu et al. reported that PTH 1-34 treatment of mouse MC-3T3-E1 osteoblastic cells also increased $\beta$-catenin protein levels, and that this effect occurred through modulation of both the protein kinase $A$ and protein kinase C pathways [49]. Furthermore, the authors showed that treatment of the cells with either PTH or $\mathrm{LiCl}_{2}$, which inhibits GSK-3 $\beta$ and activates canonical Wnt signaling, suppressed apoptosis induced by either dexamethasone or etoposide.

Collectively, these studies demonstrate that as in other cell types, Wnt signaling plays an important role in the control of bone cell apoptosis. Moreover, suppression of PCD is at least part of the mechanism by which this important pathway modulates bone formation. However, it is not yet entirely clear to what extent the effects of canonical Wnt signaling on osteoblast and osteocyte apoptosis contribute to the overall impact of this pathway on bone formation. Therefore, future studies in this area should be directed at trying to dissect out the regulation of cellular apoptosis from other aspects of osteoblast physiology.

\section{Acknowledgments}

I wish to thank all of my colleagues at Wyeth Research, as well as our academic collaborators, for their important contributions to the primary research articles and preliminary reports that were cited in this article.

\section{References}

1 Wodarz A, Nusse R. Mechanisms of Wnt signaling in development. Annu Rev Cell Dev Biol 1998;14:59-88.

2 Miller JR. The Wnts. Genome Biol 2002; 3: reviews3001.1-reviews3001.15.

3 Logan CY, Nusse R. The Wnt signaling pathway in development and disease. Annu Rev Cell Dev Biol 2004; 20:781-810.

4 Moon RT, Kohn AD, De Ferrari GV, Kaykas A. WNT and betacatenin signalling: diseases and therapies. Nat Rev Genet 2004; 5:691-701.

5 Sharpe C, Lawrence N, Martinez Arias A. Wnt signalling: a theme with nuclear variations. Bioessays 2001; 23:311-318.

6 Veeman MT, Axelrod JD, Moon RT. A second canon: functions and mechanisms of beta-catenin-independent Wnt signaling. Dev Cell 2003; 5:367-377.

7 Kuhl M, Sheldahl LC, Park M, Miller JR, Moon RT. The Wnt/ $\mathrm{Ca}^{2+}$ pathway: a new vertebrate Wnt signaling pathway takes shape. Trends Genet 2000; 16:279-283.

8 Weston CR, Davis RJ. The JNK signal transduction pathway. Curr Opin Genet Dev 2002; 12:14-21.

9 Chen AE, Ginty DD, Fan C-M. Protein kinase A signaling via CREB control myogenesis induced by Wnt proteins. Nature 2005; 433:317-322.

10 Pourquie O. A new canon. Nature 2005; 433:208-209.

11 Zorn AM. Wnt signaling: antagonistic dickkopfs. Current Biology 2001; 11:R592-R595.

12 Jones SE, Jomary C. Secreted Frizzled-related proteins: searching for relationships and patterns. Bioessays 2002; 24:811-820.

13 Kawano Y, Kypta R. Secreted antagonists of the Wnt signalling pathway. J Cell Sci 2003; 116:2627-2634.

14 Rawadi G, Roman-Roman S. Wnt signaling pathway: a new target for the treatment of osteoporosis. Expert Opin Ther Targets 2005; 9:1063-1077.

15 van Bezooijen RL, ten Dijke P, Papapoulos SE, Lowick CWGM. $\mathrm{SOST} /$ sclerostin, an osteocyte-derived negative regulator of bone formation. Cytokine Growth Factor Rev 2005; 16:319-327.

16 Hardt SE, Sadoshima J. Glycogen synthase kinase -3beta: a novel regulator of cardiac hypertrophy and development. Circ Res 2002; 90:1055-1063.

17 You L, Kim J, He B, Xu Z, McCormick F, Jablons DM. Wnt-1 signal as a potential cancer therapeutic target. Drug News Perspect 2006; 19:27-31.

18 Chen S, Guttridge DC, You Z, et al. Wnt-1 signaling inhibits apoptosis by activating beta-catenin/T cell factor-mediated transcription. J Cell Biol 2001;152:87-96.

19 Melkonyan HS, Chang WC, Shapiro JP, et al. SARPs: a family of secreted apoptosis-related proteins. Proc Natl Acad Sci USA 1997; 94:13636-13641.

20 Han X, Amar S. Secreted frizzled-related protein 1 (SFRP1) protects fibroblasts from ceramide-induced apoptosis. $J$ Biol Chem 2004; 279:2832-2840.

21 Manolagas SC. Birth and death of bone cells: basic regulatory mechanisms for the pathogenesis and treatment of osteoporosis. Endocr Rev 2000; 21:115-137.

22 Mundy GR, Chen D, Oyajobi BO. Bone remodeling. In: Favus MJ, ed. Primer on the metabolic bone diseases and disorders of mineral metabolism. American Society of Bone and Mineral Research: Washington 2003:46-58.

23 Bodine PV, Komm BS. Tissue culture models for studies of hormone and vitamin action in bone cells. Vitam Horm 2002; 64:101-151.

24 Lian JB, Stein GS, Aubin JE. Bone formation: maturation and functional activities of osteoblast lineage cells. In: Favus MJ, ed. Primer on the metabolic bone diseases and disorders of mineral metabolism. American Society for Bone and Mineral Research: Washington 2003:13-28.

25 Teitelbaum SL. Bone resorption by osteoclasts. Science 2000; 289:1504-1508.

26 Lian JB, Stein GS. Runx2/Cbfa1: a multifunctional regulator of bone formation. Curr Pharm Des 2003; 9:2677-2685.

27 Komori T. Runx2, a multifunctional transcription factor in skeletal development. J Cell Biochem 2002; 87:1-8. 
28 Boyce BF, Xing L, Jilka RL, et al. Apoptosis in bone cells. In: Bilezikian JP, Raisz LG, Rodan GA, eds. Principles of bone biology. Volume 1. Academic Press: San Diego 2002:151-168.

29 Koay MA, Brown MA. Genetic disorders of the LRP5-Wnt signalling pathway affecting the skeleton. Trends Mol Med 2005; 11:129-137.

30 Ferrari SL, Deutsch S, Antonarakis SE. Pathogenic mutations and polymorphisms in the lipoprotein receptor-related protein 5 reveal a new biological pathway for the control of bone mass. Curr Opin Lipidol 2005; 16:207-214.

31 Gong Y, Slee RB, Fukai N, et al. LDL receptor-related protein 5 (LRP5) affects bone accrual and eye development. Cell 2001; 107:513-523.

32 Patel MS, Karsenty G. Regulation of bone formation and vision by LRP5. N Engl J Med 2002; 346:1572-1574.

33 Johnson ML, Harnish K, Nusse R, Van Hul W. LRP5 and Wnt signaling: a union made for bone. J Bone Miner Res 2004; 19:1749-1757.

34 Johnson ML. The high bone mass family - the role of Wnt/LRP5 signaling in the regulation of bone mass. J Musculoskelet Neuronal Interact 2004; 4:135-138.

35 Little RD, Carulli JP, Del Mastro RG, Dupuis J, Osborne M, Folz $\mathrm{C}$, et al. A mutation in the LDL receptor-related protein 5 gene results in the autosomal dominant high-bone-mass trait. $\mathrm{Am} \mathrm{J}$ Hum Genet 2002; 70:11-19.

36 Boyden LM, Mao J, Belsky J, Mitzner L, Farhi A, Mitnick MA, et al. High bone density due to a mutation in LDL-receptor-related protein 5. N Engl J Med 2002; 346:1513-1521.

37 Johnson ML, Gong G, Kimberling W, Recker SM, Kimmel DB, Recker RR. Linkage of a gene causing high bone mass to human chromosome 11 (11q12-13). Am J Hum Genet 1997; 60:13261332.

38 Van Wesenbeeck L, Cleiren E, Gram J, Beals RK, Benichou O, Scopelliti D, et al. Six novel missense mutations in the LDL receptor-related protein 5 (LRP5) gene in different conditions with an increased bone density. Am J Hum Genet 2003; 72:763771.

39 He X, Semenov M, Tamai K, Zeng X. LDL receptor-related proteins 5 and 6 in Wnt/beta-catenin signaling: arrow points the way. Development 2004; 131:1663-1677.

40 Ellies DL, Viviano B, McCarthy J, Rey J-P, Itasaki N, Saunders $\mathrm{S}$, et al. Bone density ligand, sclerostin, directly interacts with LRP5 but not LRP5G171V to modulate Wnt activity. J Bone Miner Res 2006; 21:1738-1749.

41 Li X, Zhang Y, Kang H, Liu W, Liu P, Zhang J, et al. Sclerostin binds to LRP5/6 and antagonizes canonical Wnt signaling. J Biol Chem 2005; 280:19883-19887.

42 Semenov MV, He X. LRP5 mutations linked to high bone mass diseases cause reduced LRP5 binding and inhibition by SOST. $J$ Biol Chem 2006; 281:38276-38284.

43 Kato M, Patel MS, Levasseur R, Lobov I, Chang BH, Glass DA, 2nd, et al. Cbfa1-independent decrease in osteoblast proliferation, osteopenia, and persistent embryonic eye vascularization in mice deficient in Lrp5, a Wnt coreceptor. J Cell Biol 2002; 157:303-314.

44 Babij P, Zhao W, Small C, et al. High Bone Mass In Mice Expressing A Mutant LRP5 Gene. J Bone Miner Res 2003; 18:960974.

45 Bodine PV, Billiard J, Moran RA, et al. The Wnt antagonist secreted frizzled-related protein-1 controls osteoblast and osteocyte apoptosis. J Cell Biochem 2005; 96:1212-1230.

46 Bodine PV, Zhao W, Kharode YP, et al. The Wnt antagonist secreted frizzled-related protein-1 is a negative regulator of trabecular bone formation in adult mice. Mol Endocrinol 2004; 18:1222-1237.

47 Wang FS, Lin CL, Chen YJ, et al. Secreted frizzled-related protein 1 (SFRP1) modulates glucocorticoid attenuation of osteogenic activities and bone mass. Endocrinology 2005; 146:2415-2423.

48 Almeida M, Han L, Bellido T, Manolagas SC, Kousteni S. Wnt proteins prevent apoptosis of both uncommitted osteoblast progenitors and differentiated osteoblasts by beta-catenin-dependent and -independent signaling cascades involving Src/ERK and phosphatidylinositol 3-kinase/AKT. J Biol Chem 2005; 280:41342-41351.

49 Tobimatsu T, Kaji H, Sowa H, et al. Parathyroid hormone increases beta-catenin levels through Smad3 in mouse osteoblastic cells. Endocrinology 2006; 147:2583-2590. 